\title{
Fordern und fördern
}

\section{Matthias Scholer}

Wissenschaftsjournalist

EPAs, CBME, AbAs, Mini-Cex, DOPS - die ärztliche Aus-, Weiter- und Fortbildung ist in Bewegung. Am vierten Symposium für ärztliche Weiterbildner erhielten die Teilnehmenden nicht nur einen wertvollen Überblick über die neusten Trends und Technologien im Weiterbildungsbereich, sondern auch über aktuelle Forschungsergebnisse im Lehr- und Lernbereich.

«Was man nicht messen kann, kann man nicht lenken.» Mit diesem Zitat des Ökonomen Peter Drucker eröffnete Jürg Hodler das diesjährige Symposium für ärztliche Weiterbildner, welches unter dem Titel «Ärztinnen und Ärzte in der Weiterbildung fordern und för-

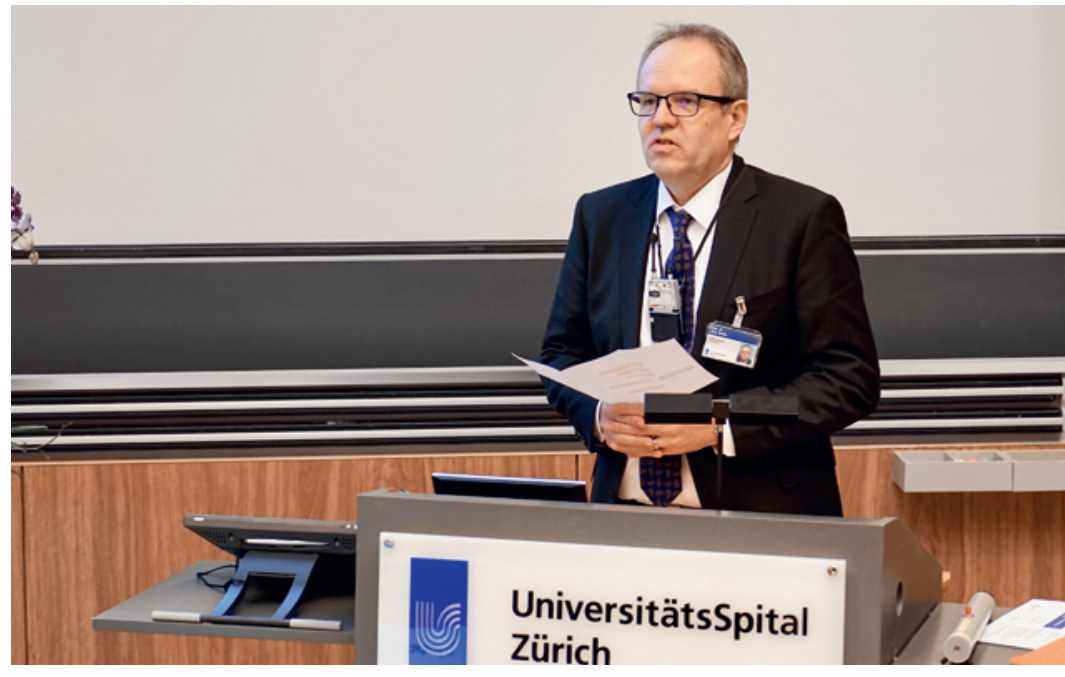

Jürg Hodler, Ärztlicher Direktor des USZ, eröffnete die Veranstaltung.

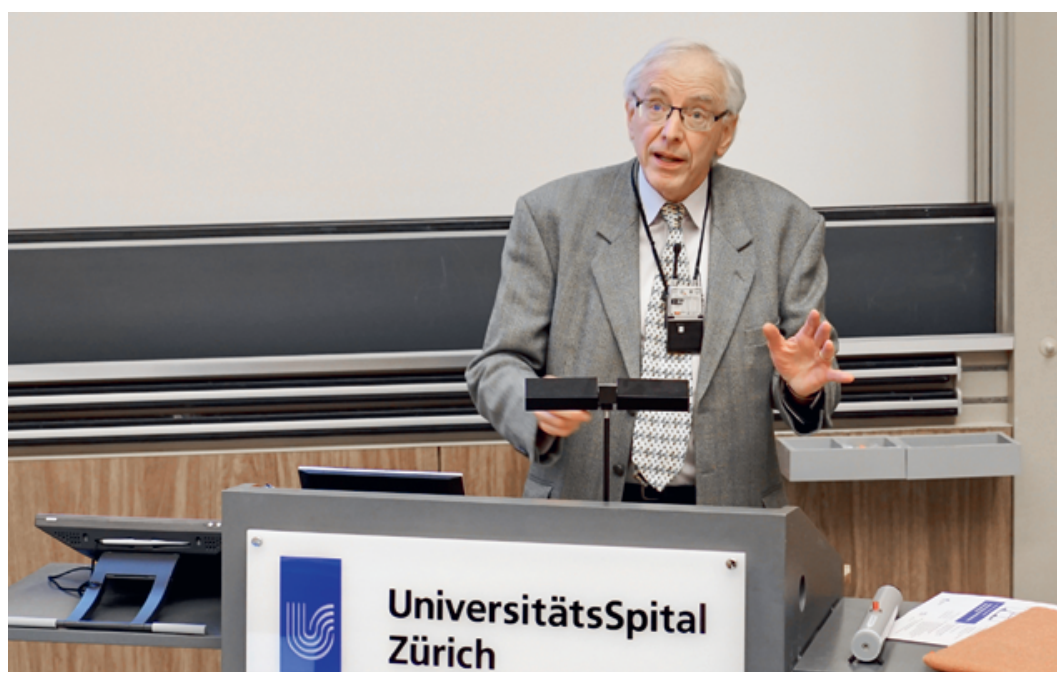

Organisator Hans Ulrich Bucher, der Delegierte für ärztliche Weiterbildung USZ. dern» stand. Hodler zeigte sich beeindruckt von den gut besetzten Zuschauerrängen an diesem Freitagnachmittag - ein untrügliches Zeichen, dass die Möglichkeiten zur Optimierung der ärztlichen Weiterbildung auf grosses Interesse stossen.

Nachdem im letzten Jahr die «Competence Based Medical Education» (CBME) das Hauptthema war, drehte sich in diesem Jahr alles um Möglichkeiten, den Wissensstand und die Fähigkeiten der Kandidatinnen und Kandidaten direkt an ihrem Arbeitsplatz möglichst präzise beurteilen zu können.

\section{Rückschläge auf der Lernkurve}

Den Anfang machte das Rednerduo Adrian Marty, Medizinischer Leiter am Simulationszentrum und Oberarzt am Institut für Anästhesiologie des Universitätsspitals Zürich (USZ), und James Crossley, der an der Universität Sheffield einen Lehrstuhl im Bereich der Medizinischen Ausbildung innehat.

Marty führte die Zuhörenden mit einer Geschichte ins Thema ein. Im deren Mittelpunkt stand, stellvertretend für unzählige Assistenzärztinnen und -ärzte, der junge Assistenzarzt Paul. Dieser ist zu Beginn seiner Assistenzzeit top motiviert. Entsprechend gross ist seine Erwartungshaltung in Bezug auf das anstehende Trainingsprogramm durch seine Ausbildner. Doch seine Lernkurve verläuft alles andere als kontinuierlich aufwärts. Sie ist durch zahlreiche Abstürze geprägt. Dies habe, so erklärte Marty, vor allem damit zu tun, dass Paul zwar kontinuierlich an Selbstvertrauen und Kompetenz zulegt, aber mit jedem Wechsel seines Vorgesetzten, und erst recht beim Wechsel der Abteilung oder der Weiterbildungsstätte, sackt das aufgebaute Vertrauen in seine Fähigkeiten massiv zusammen. Die muss er sich immer wieder aufs Neue beweisen. Zudem enthalten seine Zwischenzeugnisse bloss allgemeingültige Bemerkungen wie «Erfüllt die Erwartungen», oder «Nur weiter so». Zum Schluss sei- 


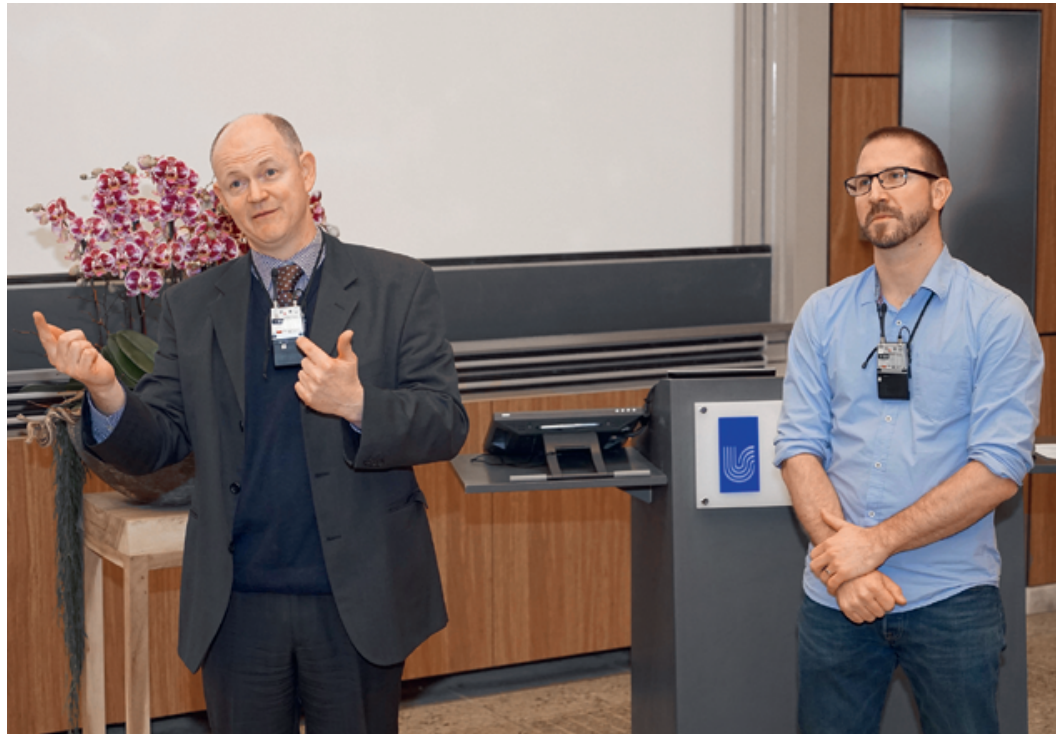

Unterhaltsames Rednerduo: James Crossley (links) und Adrian Marty.

ner Weiterbildung legt Paul dann eine Prüfung ab, die ihn eigentlich berechtigt, selbständig zu arbeiten. Nur: Paul ist sich überhaupt nicht sicher, ob er wirklich für den Praxisalltag bereit ist.

\section{Für den Praxisalltag gerüstet?}

An diesem Punkt übernahm James Crossley das Mikrofon, um die Gründe für Pauls unbefriedigende Situation und mögliche Gegenmassnahmen aufzuzeigen. Für den Briten steht fest, dass es im bestehenden System vor allem zwei Schwachstellen in der Beurteilung von Kandidatinnen und Kandidaten gibt. Erstens bilden die meisten Arbeitsplatzbasierten Assessments (AbAs) den Fortschritt eines Kandidaten kaum aussagekräftig ab. Zweitens ist eine bestandene Prüfung kein Garant, dass jemand wirklich für den Praxisalltag genügend gerüstet ist. Oder wie es Crossley mit seinem britischen Humor ausdrückte: «Viele von uns kennen jemanden, der zwar eine Prüfung bestanden hat, man aber trotzdem froh ist, dass er kein Mitglied der eigenen Familie behandelt.» Aber auch das Gegenteil ist möglich: Kandidatinnen und Kandidaten, die an einer Prüfung zwar schlecht abschneiden, sich aber im Praxisalltag kompetent zeigen. Crossley brachte die Situation, basierend auf verschiedenen wissenschaftlichen Untersuchungen, wie folgt auf den Punkt: Von einer Performance unter Prüfungsbedingungen lassen sich keine Schlüsse auf die Arbeit im Praxisalltag ziehen. Dies habe, so der Referent, vor allem damit zu tun, dass man im Alltag komplett anders funktioniere. Schliesslich arbeitet man im Alltag unter Zeitdruck, muss mehrere Dinge gleichzeitig erledigen und konstant seine Tätigkeiten priorisieren.

\section{Beurteilungen unter realen Praxis- bedingungen}

Das Problem mit einer "künstlichen Umgebung», die für ein Examen geschaffen wird, existiert aber auch bei den regelmässig stattfindenden AbAs. Wenn dafür speziell ausgesuchte Patienten der Kandidatin, dem Kandidaten zugeteilt werden, können Rahmenbedingungen entstehen, die nicht dem Alltag entsprechen. Folglich brauche es vermehrt Spontanität und «richtige Patienten", um die Arbeit und Kompetenz eines Kandidaten abschätzen zu können.

Das nächste Problem rund um die AbAs sieht James Crossley in der, meist auf Grund chronischer Arbeitsüberlastung, zeitlich stark versetzten Beurteilung einer Kandidatin, eines Kandidaten durch den zuständigen Supervisor. Damit kommt es fast zwangsläufig zu generalisierten, wenig aussagekräftigen Beurteilungen. Verstärkt wird dieser Missstand durch die Tatsache, dass es einerseits nie einfach ist, jemandem eine schlechte Beurteilung auszustellen. Andererseits zeigen die Weiterzubildenden auch nur immer ihre Stärken. Schwächen oder Unsicherheiten werden gekonnt versteckt. Ein «failure to fail» seitens der Weiterbildner und einer "fear of failure» seitens der Assistentinnen und Assistenten sei das Resultat. Crossley rät deshalb den Weiterbildnern, nicht nur zu überprüfen, ob das theoretische Wissen dem momentanen Ausbildungsstand entspricht. Vielmehr sollen sie sich auch immer die Frage stellen, ob sie einer Kandidatin, einem Kandidaten die Arbeit am Krankenbett zutrauen oder nicht. Crossley und sein Team haben die Auswirkungen dieses Vorgehens bei Chirurgen und Internisten wissenschaftlich untersucht. Mit einem eindrücklichen Resultat: Um eine Kandidatin, einen Kandidaten verlässlich und reproduzierbar einschätzen zu können, braucht es viel weniger Supervisoren im Vergleich zu den herkömmlichen Prüfungsszenarien.

Und noch einen Vorteil hat es, sich bei einer Kandidatenbeurteilung die Frage zur Vertrauenswürdigkeit zu stellen: Sollte man zum Schluss kommen, einem Kandidaten mitteilen zu müssen, dass man ihm noch keine selbständige Arbeit mit Patienten zutraut, schafft dies eine ideale Basis, um konstruktiv Schwächen ansprechen und Gegenmassnahmen einleiten zu können.

Abschliessend fasste der Brite seine Ausführungen zusammen: Einerseits können Examen in seinen Augen bloss bestätigen, dass jemand das theoretische Wissen für die Praxis hat. Damit lässt sich jedoch nicht beurteilen, ob jemand für die Praxis reif ist. Andererseits lassen sich Lernfortschritte mittels AbAs aussagekräftig beurteilen, wenn denn dabei die richtigen Fragen gestellt werden. 


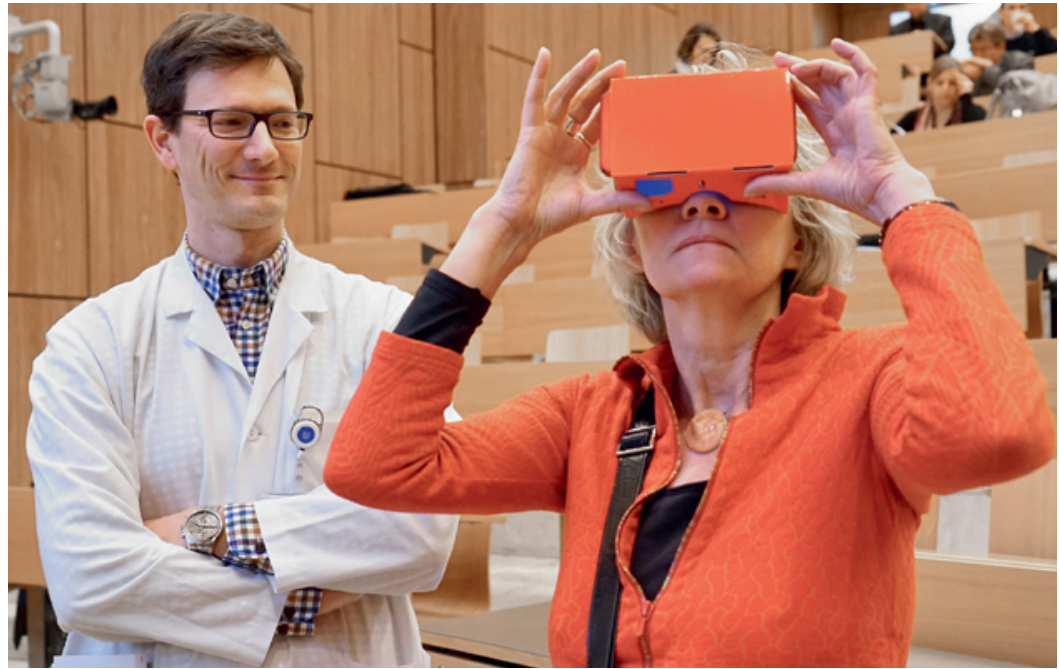

Alexander Navarini (USZ) stellte die interaktive App «Virtual Derm» vor.

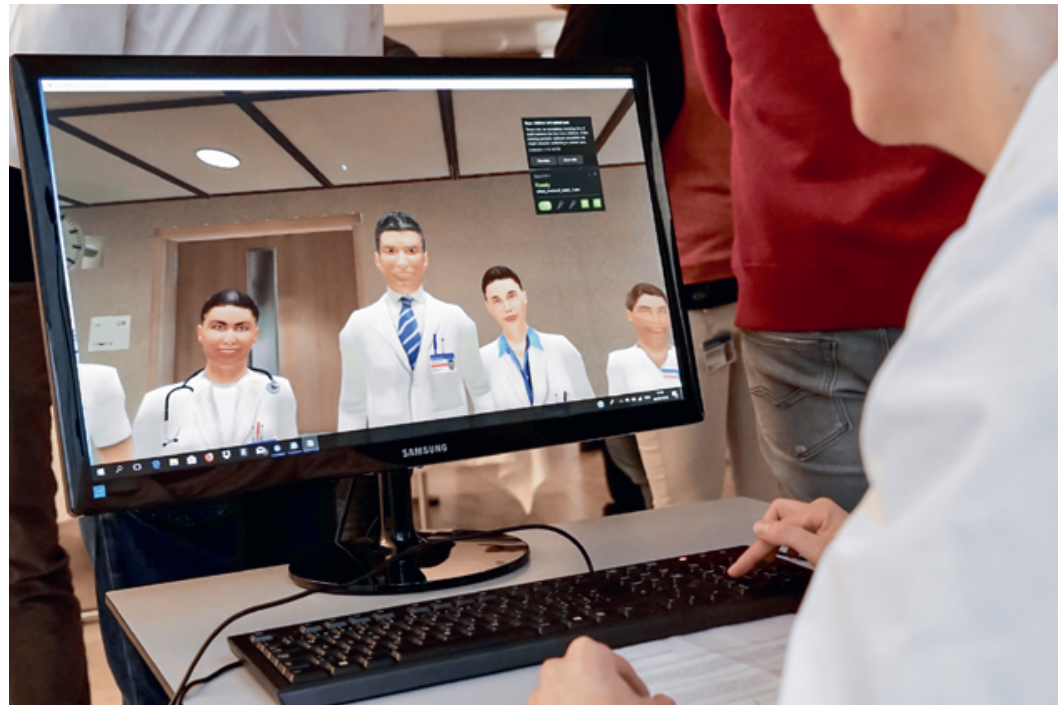

Auch die «Virtuelle Interaktive Chefvisite» konnte von den Teilnehmenden getestet werden.

Zum Abschluss dieses Teils der Veranstaltung übernahm dann Adrian Marty nochmals das Mikrofon, um aus dem Gehörten sein Fazit für den Klinikalltag zu ziehen. Marty schlägt vor, während des Arbeitsalltags kontinuierlich viele kleine Datenpunkte zu sammeln, die, wie pixelartig, erlauben, sich mit der Zeit ein präzises Gesamtbild einer Kandidatin, eines Kandidaten machen zu können. Marty und sein Team wollen die kritische Grösse an Datenpunkten bestimmen, mit der sich das Niveau eines Kandidaten objektiv und möglichst niederschwellig beurteilen lässt. Ziel muss es laut Marty sein, ein kreatives Beurteilungssystem zu entwickeln, mit denen alle Seiten gerne arbeiten. So lassen sich Rückschläge in der Lernkurve minimieren Dadurch kann die Motivation der Weiterzubildenden aufrechterhalten und die Effizienz der Weiterbildung optimiert werden.

\section{Virtual Reality}

Nach diesem vertieften Einblick in die Beurteilungskriterien der Weiterzubildenden wurden zwei Applikationen vorgestellt, welche stellvertretend für den Einsatz neuer Technologien stehen. Dabei handelt es sich einerseits um die interaktive App für angehende Dermatologen "Virtual Derm» und die "Virtuelle Interaktive Chefvisite». Die Applikation «Virtual Derm» könnte in $\mathrm{Zu}$ kunft die traditionelle 3D-Lernmethode mit Moulagen ersetzen. Dafür legen sich die Auszubildenden ihre smartphones in eine VR-Brille und klicken sich anschliessend durch eine umfangreiche Bildersammlung verschiedener dermatologischer Krankheitsbilder, zu denen sie dann entsprechende Fragen beantworten müssen. Ebenfalls eine VR-Brille ist für den Gebrauch der Virtuellen Interaktiven Chefvisite nötig. Dabei wird der User in die Situation einer Chefarztvisite versetzt, deren Verlauf beziehungsweise der Schwierigkeitsgrad der Fragen vom Coach gesteuert werden kann. Beide Applikationen standen in der Pause den Besuchern zum Testen zur Verfügung - ein Angebot, das rege genutzt wurde.

\section{Die Lehre muss aufgewertet werden}

Beim nächsten Referat standen Mini-CEX und DOPS im Mittelpunkt, wobei Mini-CEX für mini clinical examination und DOPS für direct oberservation of procedural skills steht. Zwei Formen eines arbeitsplatzbasierten Assessments, wie es in der Schweiz in der Weiterbildung für alle Fachrichtungen gefordert wird. Welches die Stolpersteine, aber auch die Erfolgsfaktoren bei Mini-CEx und DOPS sind, untersuchten Andrea Lörwald und PD Dr. Dr. med. Sören Huwendiek, beide vom Institut für Medizinische Lehre der Universität Bern. Lörwald hat sich als Postdoc eingehend mit Mini-Cex und DOPS beschäftigt, da noch einige Forschungslücken rund um die Effekte der beiden AbAs-Formen bestanden.

Die beiden Referenten zeigten zuerst die entscheidenden Stolpersteine auf: Dazu gehören unter anderem, dass für die Abas häufig zu wenig Zeitressourcen eingeplant werden, die Verantwortlichkeiten und Ziele nicht definiert sind und AbAs im Klinikalltag zu wenig ernstgenommen werden.

Als entscheidenden Erfolgsfaktor führten die beiden Wissenschaftler denn auch die Notwendigkeit, die Lehre in der Weiterbildung aufzuwerten und eine entsprechende Feedback-, Lehr- und Lernkultur in den einzelnen Institutionen zu schaffen, auf.

Wie weit man teilweise von diesen Vorgaben entfernt ist, zeigte sich in der anschliessenden Podiumsdiskussion mit dem Titel «Kunst der aufbauenden Kritik». 

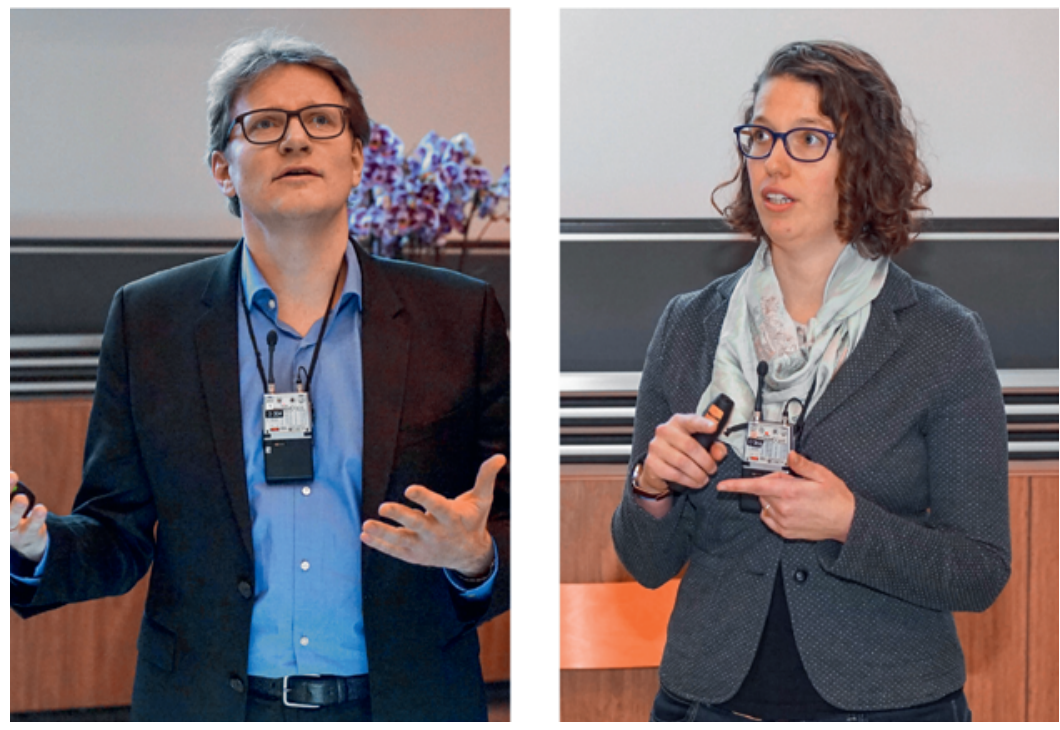

Sören Huwendiek und Andrea Lörwald (beide UniBe) untersuchten die Erfolgsfaktoren von Mini-CEX und DOPS.

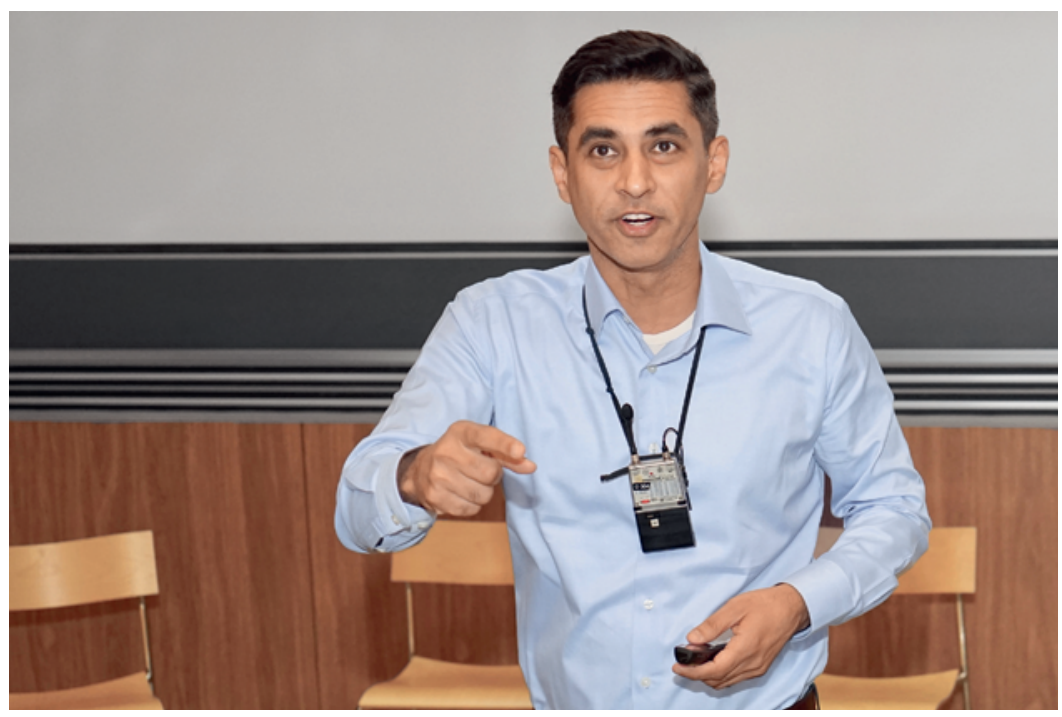

Manu Kapur plädiert für eine Verstärkung des kontextbasierten Lernens mittels interaktiver Stimulation.

In der illustren Runde, bestehend aus Vertreterinnen und Vertretern verschiedener Fachrichtungen und Hierarchiestufen, wurden eigene Erfahrungen mit den AbAs beziehungsweise der Schwierigkeit, diese im Klinikalltag sinnvoll einzubetten, diskutiert.

\section{Produktives Scheitern fördern}

Den Schlusspunkt der Veranstaltung setzte Manu mscholer[at]emh.ch schung und Leiter des Institute of Learning Sciences and Higher Education der ETH Zürich. Kapur prägte mit seinen wissenschaftlichen Arbeiten den Begriff des productive failure - dem produktiven Scheitern also. Die These, dass uns Scheitern viel weiter bringt, als ein Erfolg, konnte in zahlreichen Studien belegt werden. Eine Erkenntnis, die vieles in der derzeitigen medizinischen Grund- und Weiterbildung über den Haufen werfen könnte. So kritisiert Manu Kapur, dass an Universitäten grösstenteils noch der traditionelle Aufbau zum Einsatz kommt: Zuerst wird den Studierenden in Frontalunterreicht viel Grundwissen vermittelt. Erst in einem weiteren Schritt sollen sie das Gelernte dann in der Praxis umsetzen. In Kapurs Augen ein Fehler. Denn beim Frontalunterricht kommt Kreativität, Problemlösungskompetenz und die Fähigkeit, Gelerntes in konkreten Problemstellungen anzuwenden, zu kurz. Deshalb rät der Forscher, den Studierenden zu Beginn eines neuen Lernstoffes nicht gleich zu sagen, worum es geht. Vielmehr sollte man ihnen eine Aufgabe stellen, die sie nicht gänzlich lösen können. So erkennen sie für sich, was sie in diesem Themenbereich schon wissen und wo noch Wissenslücken bestehen. Letztere werden dann im Rahmen der Vorlesungen gefüllt - ein Vorgehen, das einen hohen Lerneffekt verspricht. An der ETH wird dieses Vorgehen schon umgesetzt. So wurden die Bachelorstudenten Humanmedizin der ETHZ bereits während der ersten Woche ins Spital geschickt mit dem Ziel, die Studierenden zu aktivieren und ihnen bewusst zu machen, welches Wissen in einigen Jahren von ihnen erwartet wird. Gut möglich also, dass kontextbasiertes Lernen mittels interaktiver Stimulation in Zukunft auch in der humanmedizinischen Aus- und Weiterbildung zum Einsatz kommt.

Dem Organisator Hans Ulrich Bucher ist es mit dem vierten Symposium erneut gelungen, ein äusserst spannendes und informatives Programm zusammenzustellen, von dem die Anwesenden alle etwas in ihren eigenen Berufsalltag mitnehmen konnten. Herr Bucher emeritiert dieses Jahr. Es ist jedoch geplant, diese Veranstaltungsreihe auch im kommenden Jahr weiterzuführen.

\section{Podcasts und Tagungsunterlagen}

Hinweis: Die aufgezeichneten Vorträge und Diskussionen und die gezeigten Präsentationen sind über folgenden Link abrufbar: www.usz.ch/epa 